\title{
Predicted responses to selection across the climatic range of a rainforest Drosophila without local adaptation: environmental variation limits trait divergence along ecological gradients
}

Authors: Eleanor K. O’Brien ${ }^{1,2^{*}}$, Megan Higgie ${ }^{3}$, Andrew D. Saxon ${ }^{1}$, Ary A. Hoffmann ${ }^{4}$ \& Jon R. Bridle ${ }^{1,5}$

\section{Author affiliations:}

1. School of Biological Sciences, Life Sciences Building, University of Bristol, Bristol BS8 1TQ, United Kingdom

2. Current address: Centre for Precision Health, School of Medical \&Health Science, Edith Cowan University, WA 6027, Australia

3. College of Marine \& Environmental Sciences, James Cook University, Queensland 4811, Australia

4. Bio21 Institute, University of Melbourne, Victoria 3052, Australia

5. Department of Genetics, Environment \& Evolution, University College London, London, United Kingdom

\section{Author email addresses:}

EKO: eleanor.obrien@bristol.ac.uk

MH: megan.higgie@jcu.edu.au

ADS: andy.saxon@bristol.ac.uk

AAH: ary@unimelb.edu.au

JRB: j.bridle@ucl.ac.uk

\section{Corresponding author:}

Eleanor K. O'Brien, School of Biological Sciences, Life Sciences Building, University of Bristol, United Kingdom; Ph: +61 467527816, Email: Eleanor.obrien@bristol.ac.uk

Running title: Limits to trait evolution in Drosophila birchii

Keywords: caged transplant experiments; Drosophila; elevation gradient; fitness; genetic variance; heritability; quantitative traits; selection

Acknowledgements: Peter Alexander, Chris Clinton, Ciara Mann and Lara Meade assisted with the field transplant experiments. Rachel Taylor, Alice Greaves, Calum Pennington and Tim Perry assisted with assays of cold and heat tolerance. Gareth Hobbs, Alexander Kelly, Laura Brooks, Laura Perkins, Holly So, Ali Somerville and Maddie Marzola assisted with mounting and measuring wings. Thank you to Greg Walter for helpful feedback on this manuscript. This work was funded by Natural Environment Research Council grant no. NE/G007039/1 to JRB. 


\section{Abstract}

Evolutionary responses to environmental change require heritable variation in traits under selection. Both heritability and selection vary with the environment, and may also covary, meaning that environmental variation can be an important source of evolutionary constraint. However, estimates of heritability and selection along environmental gradients in the field are rare. We estimated environmental variation in selection on three traits (cold tolerance, heat tolerance and wing size) of the rainforest fly Drosophila birchii by transplanting flies in cages along two elevational gradients in north-east Queensland, Australia, and calculating the genetic covariance of trait values with cage productivity at each elevation. We estimated heritability of each trait from laboratory crosses, and environmental variation in heritability of wing size from the correlation of mothers and daughters in cages at each elevation. We then used estimates of selection and heritability to predict selection responses along the elevation gradients. Laboratory assays revealed low-moderate genetic variation in all traits and low covariation among traits, suggesting the potential for a strong response to selection. Estimated selection responses predicted divergence of cold tolerance with elevation at one gradient. However, this was not observed at either gradient, with no difference between high and low elevation populations for this trait. Despite substantial variation in heritability (and predicted selection response) of wing size, this appeared random with respect to elevation, preventing overall divergence and suggesting that local environmental variation constrains evolutionary responses along natural ecological gradients. Such an effect, if widespread, may significantly slow evolutionary responses to environmental change. 


\section{Introduction}

Climate change and habitat loss are altering abiotic and biotic environmental conditions and are a major threat to global biodiversity (Sala et al. 2000; Urban 2015; Nadeau et al. 2017). Evolutionary responses to these environmental changes are likely to be crucial for the persistence of many species across their ranges, particularly where populations are unable to migrate to track suitable habitat due to geographic or biological barriers to dispersal (Bridle \& Vines 2007; Hoffmann \& Sgrò 2011). Evolutionary responses require heritable variation in trait(s) under selection, as described by the breeder's equation (Lush 1937; Falconer \& Mackay 1996) and its multivariate equivalent (Lande 1979). Quantitative genetic analyses have revealed significant heritable variation in a diverse range of traits (e.g. Mousseau \& Roff 1987; Mousseau et al. 2000; Kruuk et al. 2008), and provide examples of apparently strong selection on many traits (e.g. Kingsolver et al. 2001; Kruuk et al. 2002; Hereford et al. 2004), suggesting that the potential for adaptive evolution is high. However, evolutionary responses in nature are often weaker or even in the opposite direction to those predicted (e.g. Kruuk et al. 2002; Postma et al. 2007; Wilson et al. 2007; Gienapp et al. 2008; Pemberton 2010). Explanations for this include correlations with other (unmeasured) traits (Blows \& Hoffmann 2005; Walsh \& Blows 2009), and the effects of spatial and temporal environmental variation in the field on both heritability (Hoffmann \& Merilä 1999; Charmantier \& Garant 2005) and the strength of selection (Siepielski et al. 2013; Hayward et al. 2017; Siepielski et al. 2017). These issues mean that estimates of heritability and selection made in under 
constant conditions in the laboratory will often have minimal value in predicting evolutionary responses in the field.

Direct effects of the environment on traits (e.g. through condition dependence, or plasticity, or both) can affect their heritabilities, although it remains unclear whether heritability is increased or decreased in less favourable environments (Hoffmann \& Merilä 1999; Charmantier \& Garant 2005; Rowiński \& Rogell 2017). Selection on a trait or combination of traits can also change between environments due to a change in the relationship of the trait(s) with fitness (Siepielski et al. 2013; Hayward et al. 2017; Siepielski et al. 2017). In addition, the environment itself may create a correlation between heritability and selection, which could constrain evolution or increase its rate along particular trajectories (Wilson et al. 2006; Husby et al. 2011; Wood \& Brodie III 2016; ; although see Ramakers et al. 2018). For example, in Soay sheep (Ovis aries), heritability and selection on birthweight were shown to be negatively correlated due to dependence of both parameters on changes in the environment among years. Specifically, heritability increased but the strength of selection decreased in years when conditions were more favourable (i.e. years when lamb survival was higher). Adaptive evolution of birthweight is therefore constrained by low heritability in harsh years and by weak selection in good years (Wilson et al. 2006). By contrast, Husby et al. (2011) found a positive correlation between heritability and selection on the timing of breeding in great tits (Parus major), which may increase the evolutionary potential of this trait to ongoing climate change.

Estimates of changes in heritability and selection along environmental gradients in the field are currently scarce in animals and are almost entirely restricted to 
vertebrates (Wilson et al. 2006; Quinn et al. 2009; Husby et al. 2011; Hayward et al. 2018). However, studies across many taxonomic groups will be critical for predicting evolutionary responses to environmental change in vulnerable species (Urban et al. 2016; Nadeau et al. 2017). Tropical insects are likely to be particularly susceptible to the effects of climate change because they typically occur in relatively stable environments close to their thermal optima, making them sensitive to minor increases in temperature mean or variance (Deutsch et al. 2008; Bonebrake \& Mastradrea 2010; Logan et al. 2014; Amarasekare \& Johnson 2017; Saxon et al. 2018). In Drosophila, some tropical specialists have lower genetic variation in key traits compared with their more widespread relatives, which may limit their potential to evolve in response to environmental change (Hoffmann et al. 2003; Kellermann et al. 2006; Kellermann et al. 2009; van Heerwaarden et al. 2009). However, heritability can also vary substantially depending on the environment in which it is measured (Van Heerwaarden \& Sgrò 2014; Cocciardi et al. 2021).

Field transplant experiments offer a powerful method of testing the effect of spatial variation in the environment on evolutionary potential, by enabling trait heritabilities and selection differentials to be estimated on the same set of genotypes at different sites along an environmental gradient (e.g. Etterson 2004). O'Brien et al. (2017) used a caged transplant experiment to estimate genetic and environmental variation in fitness of the tropical rainforest specialist Drosophila birchii along elevation gradients in north Queensland, Australia. Elevation is strongly associated with climate variables, including mean, minimum and maximum temperature and humidity, and with the abundance and fitness of $D$. birchii, making this an ecologically relevant test of how evolutionary potential is likely to be affected by 
climatic change. Fitness of $D$. birchii in cages (measured as the number of offspring produced per cage) declined significantly with increasing elevation, despite the field density of $D$. birchii being higher at high than low elevation (O'Brien et al. 2017). This suggests that when the effects of climate are considered without concomitant effects on food availability or biotic interactions, high elevation habitats are less productive than low elevation habitat, and that selection may vary in strength or direction with elevation for traits such as thermal tolerances. They also detected genetic variation in fitness in field cages, and evidence for genetic divergence in productivity when isofemale lines sourced from elevational extremes were raised under laboratory conditions. However, there was no evidence for local adaptation of $D$. birchii, even when comparing flies that had been reciprocally transplanted to the elevational limits of their distribution (O'Brien et al. 2017).

In this paper, we extend analysis of this transplant experiment to assess selection and heritability of ecologically relevant traits of $D$. birchii at sites along two elevation gradients that span the climatic range of this species. We assayed relatives of the flies transplanted in field cages for three traits expected to be under selection along these elevation gradients: cold tolerance, heat tolerance and body size. We estimated the genetic (co)variances within and between these traits and used the covariance of trait values (measured in the laboratory) with fitness of the same lines in cages in the field to estimate selection on each trait at each elevation. For body size, we also tested whether heritability varies with environment, by estimating the heritability of this trait at each elevation using a regression of the body size of daughters emerging from field cages on that of their laboratory-reared mothers. We then used our estimates of selection and heritability and applied the breeders' 
equation to predict the direction and magnitude of the evolutionary response in each trait at sites along each elevation gradient. We compared these predicted responses with trait divergence between populations from elevational extremes, to determine whether they are realised in the field.

\section{Methods}

Collection and establishment of $\mathrm{D}$. birchii isofemale lines for quantitative trait assays and field transplant experiments

We established isofemale lines (hereafter 'lines') of Drosophila birchii from fieldmated females collected from banana baits in rainforest at sites along two elevation gradients: Mt Edith (17 $\left.7.9^{\prime} \mathrm{S}, 145^{\circ} 37.2^{\prime} \mathrm{E}\right)$ and Paluma (18 $\left.59.0^{\prime} \mathrm{S}, 146^{\circ} 14.0^{\prime} \mathrm{E}\right)$ in north Queensland, Australia, using the same methods described in Bridle et al. (2009) and O'Brien et al. (2017). We established five D. birchii lines from each of eight populations: two high and two low elevation populations from each of the two gradients. These gradients are characterised by a warmer, drier, more fluctuating environment at low elevations, and a cooler, wetter, more stable environment at high elevations (see O'Brien et al. 2017 and Table S1 for temperature and humidity data at each site). Our sampling represents the full elevational range of this species at these latitudes $(0-1100 \mathrm{~m}$ above sea level), therefore it should capture the full range of phenotypic and genetic diversity in traits associated with fitness under these different climatic conditions. The lines from each population were crossed with one another (see "line cross design" below and O'Brien et al. (2017)) to generate flies for use in field caged transplants, and for estimates of thermal tolerances and body size. 
Lines maintained in the laboratory were held at $25^{\circ} \mathrm{C}$ on a $12: 12 \mathrm{hr}$ light:dark cycle unless otherwise stated.

Line cross design

We established fully reciprocal diallel crosses among lines from within each of the eight source populations from Mt Edith and Mt Paluma. This crossing design is described in detail in O'Brien et al. (2017) and is summarised briefly here. All crosses were between lines within a given source population. We paired flies from each of the five lines in every combination, excluding within-line crosses, giving 20 combinations per population. Each cross was set up by placing one virgin male and female in a vial with $10 \mathrm{ml}$ standard Drosophila media (agar, sugar, inactivated yeast, nipagin and propionic acid) for 4 days, then discarding them and leaving their offspring to emerge. This laying period results in a low density of offspring in each vial, thereby minimising effects of larval competition on traits. The crosses were set up in two separate generations: the first were established after lines had been in the laboratory for three generations, and offspring of these crosses were used in the field cage transplant experiment (see below and O'Brien et al. (2017)) and in assays of body size. The second set of crosses was established after lines had been in laboratory culture for six generations, and offspring of these crosses were used in assays of cold tolerance and heat tolerance. We established two replicate crosses per line combination for the first set of crosses, and three replicates for the second set. 
Offspring emerging from the crossing design were sexed on the day of emergence under light $\mathrm{CO}_{2}$ anaesthesia, and held in single sex vials (maximum of 10 flies per vial) until they were used in either caged transplant experiments or trait assays.

\section{Field cage transplant experiment}

In May 2012, 5910 flies emerging from the line crosses were transplanted in 591 cages to 19 sites along the two elevational gradients from which they were collected (266 cages across 10 sites at Paluma, and 325 cages across 9 sites at Mt Edith; Table S1). The details of the experimental design are described in full in O'Brien et al. (2017) and are summarised briefly below.

The transplant sites spanned the full elevation range of this species and included the low and high elevation sites from where lines originated. Because it was not feasible to replicate every line-cross combination at every site, offspring of crosses from the same maternal isofemale line were combined prior to cage establishment, by taking equal numbers of offspring from each cross. Some maternal lines failed to produce sufficient offspring to use in the cage transplant experiment. In total we transplanted 15 lines at Mt Edith and 20 lines at Paluma. All lines were transplanted to gradient ends (i.e. the two high and two low elevation sites at each gradient from which lines were sourced), but due to low productivity of some lines, not every line was transplanted at all intermediate sites. Each cage contained five virgin males and five virgin females (all aged 3 - 10 days) from the same maternal line, and each maternal line was represented by $2-4$ cages per site. Lines were only transplanted back into their gradient of origin, not between gradients, and because flies were placed in 
cages as virgins, all courtship, mating and egg-laying took place under field conditions. Survival and productivity (proxies for fitness) of flies in field cages was recorded. Analyses of variation in fitness along gradients have been published previously (O'Brien et al. 2017). Here, we assayed heat tolerance, cold tolerance and wing size on descendents of flies transplanted in field cages and used productivity in cages to estimate selection on these traits at each transplant site (see below).

Laboratory assays of quantitative traits

Using flies emerging from crosses between the same $D$. birchii linesthat were transplanted into cages in the field experiment, we assayed three traits: cold tolerance, heat tolerance and wing size. For wing size, these were the same set of crosses used to generate flies for the transplant experiment. For cold tolerance and heat tolerance, crosses between the same sets of lines were established three generations after the transplant experiment. Similar traits show repeated genetic divergence along thermal (latitudinal and elevational) gradients in several Drosophila species (e.g. Hallas et al. 2002; Hoffmann et al. 2002; Sørensen et al. 2005), including cold tolerance in D. birchii (Bridle et al. 2009).

Cold tolerance was measured as the productivity (number of offspring emerging from three days of laying) of six-day old virgin females subject to a cold shock that induced a chill coma, then mated to a single male; heat tolerance was measured on seven-day old virgin females as the time to lose standing ability on exposure to a static heat shock of $37^{\circ} \mathrm{C}$; wing size was measured as the centroid size of the right 
wing of female flies that were at least two days of age, calculated from the positions of 10 landmarks (Figure S1; Griffiths et al. (2005)). See Supplementary Note 1 for a detailed description of how trait assays were conducted.

\section{Data analysis}

All analyses were run using R v. 3.4.2 (R Core Team 2020), executed in R Studio v. 1.4.1103 (RStudio Team 2021). Details of specific packages and functions used are given in the description of each analysis.

Divergence of traits within and between elevation gradients

We tested for divergence of traits (cold tolerance, heat tolerance and body size) of $D$. birchii between the two elevation gradients (Mt Edith and Paluma) and between high and low elevation source populations within each gradient. We fitted linear mixed models using Ime4 v. 1.1-23 (Bates et al. 2015). Separate models were fitted for each trait. Gradient, elevation and their interaction were all fitted as fixed effects. Rearing vial was fitted as a random effect for all traits and batch was additionally included as a random effect in analysis of heat tolerance. The significance of fixed effects was determined using $F$-tests, and of random effects using a $x^{2}$ test to compare the log likelihood of models with and without the factor included. 
We estimated additive genetic (co)variances of the three traits using an animal model approach (Kruuk 2004; Wilson et al. 2010), implemented using MCMCglmm (v. 2.24) (Hadfield 2010). We fitted the following linear model for each gradient:

$y_{i}=\mu+A_{i}+M_{i}+e_{i}$

Where $y_{i}$ is the multivariate phenotype of individual $i, \mu$ is the multivariate phenotypic mean, $A_{i}$ is the additive genetic effect (fitted as a random effect), $M_{i}$ is the maternal effect variance (variance among mothers, beyond that explained by additive effects, fitted as a random effect) and $e_{i}$ is the residual (error) variance. Values of each trait were standardised within each gradient to a mean of 0 and a standard deviation of 1 . Standardisation was performed because large differences between traits in their means and variances can bias estimates of covariance (Hansen \& Houle 2008; Careau et al. 2015). Additive genetic effects were fitted as a matrix of predicted additive covariances between all individuals (i.e. a matrix of the pairwise coefficients of relatedness between individuals). Relatedness was estimated from a pedigree constructed based on our breeding design. See Supplementary Information (Supplementary Note 2) for a full description of the assumptions made in pedigree construction. Our data set was not large enough to estimate additive genetic (co)variances separately for each source elevation or population, but we did initially run models with source population included as a fixed factor, to account for differences in trait means between populations. However, this had no effect on (co)variance estimates, and so we present results from the simpler models run without this parameter. 
We used weakly informative inverse-Wishart priors, where the scale parameter $V$ was a $3 \times 3$ matrix, with phenotypic variance in each trait equally distributed between additive genetic, maternal effect and residual variation, and the scale parameter $n u$ equal to the number of traits. We constrained the residual covariances among traits to be 0 , since traits were measured on different individuals. We ran models for $2 \mathrm{x}$ $10^{6}$ iterations, with a burn-in of $5 \times 10^{5}$ iterations and a thinning interval of 1500 , giving a total sample size of 1000 . This resulted in autocorrelation of successive runs below 0.1 and effective sample sizes close to the maximum for all parameter estimates. Model performance was also evaluated by examining plots of the time series of the values obtained for the posterior probability distribution, and the density of this distribution for all parameters estimated from each model.

We obtained the additive genetic (co)variances of each trait and pairwise trait combination from the mode of the posterior probability distribution for the relevant model term. We calculated heritability $\left(h^{2}\right)$ as the additive genetic variance $\left(V_{\mathrm{A}}\right)$ as a proportion of total phenotypic variance $\left(V_{\mathrm{P}}\right)$. We converted genetic covariances to correlations using the posterior.cor function in MCMCglmm. We obtained the Highest Posterior Density (HPD) interval around each parameter estimate, and values derived from model parameter estimates. This defines the bounds of the region of the posterior distribution where $95 \%$ of samples lie. Additive genetic covariances and correlations between traits were deemed significant if the HPD interval did not overlap zero. Variances are constrained to be above zero, meaning that this method cannot test the significance of additive genetic variances, however we can test whether they differ significantly between gradients or traits by testing for overlap of the HPD intervals. 
Spatially variable selection on thermal tolerance and body size of $\mathrm{D}$. birchii along elevation gradients

We estimated selection differentials $(S)$ for cold tolerance, heat tolerance and body size from a regression of standardised trait values for each line estimated from laboratory assays against relative fitness in cages at each site. Separate models were fitted for each transplant site, using the Imer function in Ime4 v. 1.1-23 (Bates et al. 2015), with maternal isofemale line included as a random factor. We also fitted overall models for each gradient (using fitness at all transplant sites), which additionally included transplant site as a fixed factor. Using standardised phenotypes rather than the raw data means that $S$ is comparable across traits and between sites and gradients (Kingsolver et al. 2001).

Fitness was measured as the number of offspring emerging from each cage (see O'Brien et al. 2017) and converted to relative fitness by dividing this by the mean number of offspring produced in all cages at that transplant site (Linnen \& Hoekstra 2009). $S$ therefore gives the change in relative fitness expected with an increase of one standard deviation in the value of a given trait.

To test whether selection is expected to drive trait divergence with elevation, we ran linear models regressing estimated selection differentials on elevation for each trait at each gradient using the Im function in $\mathrm{R}$. 
Change in heritability of body size along elevation gradients

We obtained site-specific estimates of heritability of body size along the two elevation gradients, by regressing the mean body size of female offspring emerging from field cages on that of their surviving mothers (reared in the laboratory and then placed in cages to reproduce) for cages at each transplant site at Mt Edith and Paluma. For each site and gradient, we fitted linear models using the Imer function in Ime4 v. 1.1-23 (Bates et al. 2015), with maternal isofemale line as a random effect.

Mean body size of the offspring developing in cages was, on average, $7.5 \%$ larger than that of those emerging from laboratory crosses (i.e. the parental generation), and the body size of flies emerging from cages declined with increasing elevation (Figure S2). To prevent differences in trait means between generations from skewing covariance estimates (Hansen \& Houle 2008; Careau et al. 2015), we first standardised body size data within each group (parents and offspring) to a mean of 0 and a standard deviation of 1 within each transplant site.

To test whether heritability changes with elevation (as a proxy for environmental stress), we ran linear models that regressed field heritability of wing size against elevation at each gradient. We also regressed field heritability of wing size against the selection differential for this trait within each gradient, to test for environmental covariation of heritability and selection. Both of these regression analyses were conducted using the Im function in R. 


\section{Estimating response to selection along elevation gradients}

To estimate the response $(R)$ to selection on each trait at each elevation, we applied the breeders' equation:

$$
R=h^{2} S
$$

Where $S$ was the site-specific estimate of the selection differential, and $h^{2}$ was the estimated heritability of the trait. For all traits, we used the mean $h^{2}$ estimated from the laboratory crosses. This was a single value for each gradient and so ignores any environmental effect on $h^{2}$. For body size, we additionally calculated $R$ at each elevation using the site-specific estimates of $h^{2}$ from the regressions of body size of mothers and daughters in cages at each site. This allowed us to evaluate the effect of environmental variation in $h^{2}$ (as well as in $S$ ) on responses to selection along elevation gradients for this trait. To estimate errors around $R$, we took the upper and lower confidence intervals around $S$ and $h^{2}$ and inserted these into the breeders' equation in every combination. From these, we took the highest and lowest estimates of $R$ as the bounds of this estimate.

\section{Results}

Genetic divergence of thermal tolerance and body size between, but not within, elevation gradients

D. birchii lines from Mt Edith had higher cold tolerance (measured as higher fecundity following a cold-induced coma) and larger body size (wing size) than those from Paluma (Figure 1, Table 1) when reared in the laboratory. However, none of 
the three traits differed significantly between elevations within either gradient (Figure 1, Table 1).

Additive genetic (co)variances of thermal tolerance and body size from the laboratory crossing design

Heritabilities of all traits estimated from the laboratory crossing design ranged from $0.09-0.19$ (Table 2). We did not detect significant differences in heritabilities between traits, or between the two gradients, with heritability estimates having overlapping 95\% HPD intervals in all cases (Table 2). Maternal effect variance ranged from $0.09-0.15$, and also did not differ between traits or gradients (Table 2).

We did not detect significant additive genetic correlations $\left(r_{\mathrm{G}}\right)$ between any of the pairwise trait combinations at either gradient (HPD intervals all overlapped with zero; Table 3). However, these intervals were all extremely wide. Large sampling variance is common when estimating genetic covariances and correlations, meaning very large sample sizes are typically required, especially where trait correlations are weak (Visscher 1998; Bijma \& Bastiaansen 2014). Maternal effect correlations $\left(r_{\mathrm{M}}\right)$ between all pairs of traits were also indistinguishable from zero and had similarly wide confidence intervals (Table 2).

Spatially variable selection on thermal tolerance and body size of $\mathrm{D}$. birchii along elevation gradients 
When calculated overall for each gradient, selection differentials were relatively weak $(|S|=0.004-0.15)$, and non-significant in all cases (Table S2). Although none of the individual selection differentials was significantly different from zero after correction for multiple comparisons (Table S1), there was a strong and significant linear association between $S$ and elevation for cold tolerance at Paluma $\left(R^{2}=0.691\right.$, $F_{1,8}=17.9, P=0.0029 ;$ Figure 2$)$. This association was negative, implying selection for lower values of this trait at higher elevation sites (Figure 2). None of the other trait-gradient combinations showed a significant linear association between $S$ and elevation.

Change in heritability of body size along elevation gradients, estimated from correlation of laboratory-reared mothers and their field-reared daughters

Body size heritabilities estimated from mother-offspring regressions at each transplant site were similar at Mt Edith (overall $h^{2}=0.151(\mathrm{SE}=0.08)$; Table S3), but much higher at Paluma (overall $h^{2}=0.582(\mathrm{SE}=0.49)$; Table S3), compared with those estimated from the laboratory quantitative genetic breeding design (Mt Edith $h^{2}$ $=0.156$, Paluma $h^{2}=0.193 ;$ Table 2$)$. Individual site estimates exceeded lab estimates of $h^{2}$ at most sites (compare with dashed lines in Figure 3). There was however substantial variation in $h^{2}$ within each gradient, even between adjacent sites, with field-estimated $h^{2}$ ranging from $0-0.7$ at Mt Edith and $0-1$ at Paluma (Table S3, Figure 3). We did not detect a linear relationship between heritability and elevation at either gradient (Mt Edith: $R^{2}=0.008, F_{1,5}=0.039, P=0.85$; Paluma: $R^{2}$ $\left.=0.114, F_{1,8}=1.029, P=0.34\right)$. At both gradients, the highest heritabilities were seen at the elevational extremes: $h^{2}$ at the highest and lowest elevation sites at 
Paluma were higher than at other sites along the gradient $\left(h^{2} \geq 0.9\right)$ and were both highly significant $(\mathrm{P} \leq 0.01)$ (Figure 3, Table S3). At Mt Edith, none of the individual site $h^{2}$ estimates was significant, although the two highest estimates were at the lowest and second-highest elevation sites (Figure 3, Table S3). There was no evidence for covariation of field heritability and selection on wing size at either gradient (Mt Edith: $R^{2}=0.083, F_{1,5}=0.455, P=0.53$; Paluma: $R^{2}=0.158, F_{1,8}=$ $1.495, P=0.256)$.

Predicted response to selection along elevation gradients

The predicted response to selection on each trait (expressed in standard deviations (SDs) per generation) at each site along the two elevation gradients, calculated using the breeders' equation, is shown in Figure 4. For cold and heat tolerance, only a single estimate of heritability was available for each gradient (calculated from the laboratory crossing design; Table 2), therefore the selection response along the elevation gradients for each of these traits (and for wing size when using lab estimates of heritability) shows the same pattern as the selection differentials (Figure 2; Table S2). Once again, cold tolerance at Paluma is the only case where selection is predicted to drive divergence of the trait with elevation, with lower values of cold tolerance predicted at higher elevation (Figure 4).

For wing size, where we also had site-specific estimates of heritability (from regressions of mothers and daughters in cages; Figure 3, Table S3), the predicted response at most sites was the same or greater (but with wider errors) when using field $h^{2}$ estimates compared with lab $h^{2}$ estimates, particularly at Paluma (Figure 4). 
There was still no significant linear relationship between elevation and selection response when using field $h^{2}$ estimates (Mt Edith: $R^{2}=0.003, F_{1,5}=0.017, P=$ 0.901; Paluma: $\left.R^{2}=0.008, F_{1,8}=0.065, P=0.806\right)$.

\section{Discussion}

Understanding how the environment affects both selection on and heritability of traits in natural populations is critical for predicting the rate and direction of evolutionary responses to environmental change (Husby et al. 2011; Hayward et al. 2018;

Ramakers et al. 2018). We quantified variation in three ecologically important traits (cold tolerance, heat tolerance and wing size) of the rainforest fly Drosophila birchii and tested for an association between mean trait values and fitness of the same families transplanted as virgins in cages to sites along two elevation gradients, to test how the direction and strength of selection on these traits varies with environment. For all three traits we used the laboratory trait assays to estimate heritability for a given trait and genetic correlations between traits. In addition, for wing size, we could obtain an elevation-specific estimate of heritability for each site along each gradient, by regressing the wing size of females emerging from field cages against that of their laboratory-reared mothers. For this trait therefore, we were able to compare the predicted selection response at sites along each gradient calculated using a single laboratory estimate of heritability (i.e. one that doesn't take into account any effect of environment on heritability) to an estimate using elevationspecific estimates of heritability.

Two of our results in particular provide important insights into factors that may limit evolutionary responses to environmental change: 
(1) For cold tolerance at Paluma, selection differentials, and the predicted response to selection, varied with elevation in a way that should drive divergence between $D$. birchii populations at elevational extremes (Figure 2, Figure 4). However, we did not observe divergence of this trait when we compared cold tolerance of $D$. birchii sourced from populations at these extremes, suggesting that the trait variation we see in the laboratory is not visible to selection in the field.

(2) For wing size, where we had elevation-specific estimates of both selection and heritability, we found that the magnitude of predicted selection responses was typically greater when using field estimates (rather than lab estimates) of heritability. However, predicted responses varied in magnitude and direction, even between nearby sites, and as a result overall divergence of this trait with elevation was neither predicted nor observed.

Predicted response to selection on ecologically important traits of $\mathrm{D}$. birchii along elevation gradients

Our predicted selection responses would not drive divergence of heat tolerance or wing size of $D$. birchii along these elevation gradients. However, they did suggest that selection should drive lower cold tolerance of $D$. birchii with increasing elevation, at least at the Paluma gradient (although there was also a non-significant trend in the same direction at Mt Edith; Figure 4). Such lower values of cold tolerance being favoured at the colder end of a species' range seems counterintuitive, perhaps suggesting strong correlations with (unmeasured) traits that have a bigger effect on 
fitness at high elevations. Alternatively, the method used to assay this trait was reproductive output immediately following a cold shock, and delaying reproduction after an acute stress has been found to be adaptive in some cases (e.g. Wessels et al. 2011). Suppression of reproduction following a severe cold shock may therefore be favoured in populations that are periodically exposed to cold stress. Divergence of cold tolerance with elevation has previously been observed in $D$. birchii (using a different assay method; Bridle et al. 2009), demonstrating that this trait is strongly associated with fitness and does respond to selection in some cases.

Evolutionary responses may be constrained by a lack of additive genetic variation within the particular environmental context where selection is acting (e.g.

Charmantier \& Garant 2005; Rowiński \& Rogell 2017), by environmental covariance of selection and genetic variance (Wilson et al. 2006; Husby et al. 2011; Wood \& Brodie III 2016) , or if selection is weak relative to gene flow (Hendry et al. 2002a; Lenormand 2002). Furthermore, there may be other components of the environment that affect the strength of selection in ways that are not represented by our field experiment. We consider each of these factors below.

\section{Environmental variation in heritability}

Environmental variance in heritability has been shown for a wide range of taxa and traits, suggesting that the environmental context is critical for determining the potential for an evolutionary response (Kruuk et al. 2008). Unfortunately, we could not estimate environment-specific heritabilities of cold (or heat) tolerance in our field 
experiment, because these traits must be assayed on live flies, and we needed to leave flies in cages to obtain measurements of field fitness (O'Brien et al. 2017). However, we were able to assess environmental variation in heritability for wing size, which we observed was on average higher than in the lab, but showed substantial variation between sites (Figure 3, Table S3). This comparison therefore suggests that environmental variation in heritability among sites may limit evolutionary responses in this system, even without any additional effects of gene flow along the gradient (see below).

A fundamental question in evolutionary biology is whether heritability increases or decreases in stressful conditions (Hoffmann \& Merilä 1999; Charmantier \& Garant 2005; Wood \& Brodie III 2015; Rowiński \& Rogell 2017), with studies showing mixed results. For example, in $D$. birchii, additive genetic variance and heritability of desiccation resistance are much greater when the trait is assayed under less stressful conditions (Van Heerwaarden \& Sgrò 2014). By contrast, Holman and Jacomb (2017) found increased additive genetic variance under nutritional stress for morphological traits of Tribolium castaneum flour beetles. In a meta-analysis of animal studies, Rowiński and Rogell (2017) found that additive genetic variance was higher in more stressful environments for life history traits, but not morphological traits.

In our field transplant experiment, fitness of $D$. birchii in cages declined significantly with increasing elevation, both in terms of the number of offspring produced (O'Brien et al. 2017), and offspring size (Figure S2), suggesting that environmental stress increases with elevation, at least when flies are confined within cages. We did not 
observe an association between wing size heritability and environmental stress, with no significant difference between wing size heritabilities estimated at elevational extremes, and no evidence for a linear relationship of heritability with elevation. However, we did find increased heritability of wing size at the elevational extremes relative to that seen in the centre of the gradient (Figure 3; Table S3). The parentoffspring correlation used to estimate body size heritability along gradients could not separate additive genetic from maternal effects, potentially leading to overestimation of heritability (Kruuk 2004; Wilson et al. 2005). This was a constraint imposed by the design of our transplant experiment and may partially explain why parent-offspring estimates were usually higher in the field than those estimated in the laboratory using the animal model approach.

\section{Environmental covariance of selection and heritability}

Environmental coupling between selection and heritability may affect evolutionary responses to environmental change, although empirical tests of this are rare (Wilson et al. 2006; Husby et al. 2011; Wood \& Brodie III 2016; Ramakers et al. 2018). In addition, it is hard to predict the direction of any such covariance, because although the strength of selection is expected to increase in more stressful environments (Endler 1986), the effect of stress on additive genetic variance and heritability seems highly variable, as discussed above. Previous studies have shown both a positive (Husby et al. 2011) and negative (Wilson et al. 2006) covariance of heritability and selection, with opposite consequences for the expected rate of evolutionary change. We did not detect any environmental covariance of heritability and selection in wing 
size, which was the only trait for which we could obtain environment-specific heritability estimates. However, we also did not see variation in the strength of selection on this trait across the elevation gradients. For cold tolerance, we did see an increase in the strength of selection towards higher elevations, so we cannot rule out a negative covariance between heritability and selection. This trait therefore provides a potentially useful test of the importance of a covariance between heritability and selection in constraining adaptive responses, which could be investigated in a future study.

Gene flow and temporal fluctuations relative to spatial variation in selection along elevation gradients

The extent to which spatial variation in selection can drive genetic divergence of traits along a gradient also depends on the strength of selection relative to gene flow (Hendry et al. 2002b; Lenormand 2002; Bridle \& Vines 2007). Gene flow at this scale (between sites $1-10 \mathrm{~km}$ apart) is likely to be high in $D$. birchii, given very low differentiation observed at microsatellite markers between populations separated by hundreds of kilometres (Schiffer et al. 2007). The effects of spatial variation in selection on allele frequencies at such a local scale may therefore be small relative to the effect of gene flow between sites, particularly where gene flow is asymmetrical. Furthermore, our selection estimates are based on fitness estimated over a single generation. However, responses to selection in natural populations also need to account for temporal fluctuations in the strength (and possibly direction) of 
selection across generations, which may also constrain divergence along ecological gradients (Siepielski et al. 2009).

\section{Environmental determinants of selection}

Other components of the environment are also likely to drive selection in this system, even in field transplants. In particular, under natural conditions there are likely to be selective forces acting that are not captured by confining flies and their offspring to cages as we have, by necessity, done here. Although the field abundance of $D$. birchii declines towards both the warm, low elevation, and cool, high elevation ends of its range, fitness in cages always increased towards warmer sites (O'Brien et al. 2017). This discrepancy may be due to intra- and interspecific biotic interactions (which were mostly absent from cages) at low elevations (O'Brien et al. 2017), consistent with the hypothesis that species interactions are more important in limiting species' distributions at lower latitudes and elevations (Darwin 1859; Louthan et al. 2015; Paquette \& Hargreaves 2021). This result is also supported by extensive assays of the effect of biotic interactions within cages on fitness of $D$. birchii at different elevations (O'Brien et al. 2020), as well as interactions with parasitoids or pathogens, which may increase at lower elevations (Jeffs et al. 2021). Trade-offs between resistance to abiotic and biotic environmental variables may therefore limit adaptation to differing degrees along these gradients. By confining flies to cages (albeit cages located in the shade), we also limit their ability to move to suitable microclimates, or to actively forage for mates or oviposition sites, all of which are behaviours that are highly sophisticated in Drosophila (e.g. Churchill et al. 2020; 
Malek \& Long 2020; Churchill et al. 2021). These behaviours should reduce variation in the strength of selection across the gradient, therefore the cline in selection on cold tolerance that we detect may be less pronounced under natural conditions.

Increased temperature mean and variability during development has also been shown to have a detrimental effect on male (Saxon et al. 2018) and female (Cocciardi et al. 2021) reproductive success in D. birchii. We did not measure reproductive success of the flies that developed in the field cages, therefore this provides another potential explanation for the lower abundance of $D$. birchii at warmer, more variable low elevation sites in nature. If these factors (biotic interactions and developmental conditions) are key selective agents at warmer low elevation sites, they would also change our estimates of selection (and potentially heritability), and therefore predicted evolutionary responses.

\section{Limitations of our experimental design}

The number of $D$. birchii lines transplanted in cages at each site was relatively small (15-20 lines). This limited the power of our analyses to estimate selection strength at each site, which used the covariance of trait means and field productivity of lines.

Furthermore, our crossing design means that there was likely considerable withinline variation in both traits and productivity. None of our site-specific selection differentials was significantly different from zero, and it is likely that both the small number of lines and large within-line variation are contributing factors to this. 
However, while larger sample sizes would provide more precise estimates of sitespecific selection differentials, we note that the observed increase in selection strength on cold tolerance with elevation (particularly along the Paluma gradient) cannot be explained by these limitations. Therefore, by estimating this parameter at a large number of sites across an ecological gradient, we were able to uncover potentially important spatial variation in selection, even at the cost of reduced precision of site-specific estimates.

Summary and conclusions

Our assays of field fitness in relation to lab and field heritability in $D$. birchii reveal that: (1) selection in the field can be relatively weak and does not always predict trait divergence even between the extreme ends of species' climatic ranges; and (2) heritabilities in the field are also highly variable and may not be well-predicted by estimates of additive genetic variance in the laboratory. In the case of $D$. birchii, such issues make long-term responses to selection (and potentially, evolutionary rescue in response to changing conditions) difficult to predict, even from extensive field experiments.

We believe that our unique study design, which allowed us to estimate variation in both heritability and selection across the full climatic range of our study species, could provide a useful framework for addressing questions about what constrains evolution in natural systems.

\section{References}


Amarasekare, P. \& Johnson, C. (2017). Evolution of thermal reaction norms in seasonally varying environments. The American Naturalist, 189, E31 - E45.

Bates, D., Mächler, M., Bolker, B. \& Walker, S. (2015). Fitting linear mixed-effects models using lme4. Journal of Statistical Software, 67, 1-48.

Bijma, P. \& Bastiaansen, J.W.M. (2014). Standard error of the genetic correlation: how much data do we need to estimate a purebred-crossbred genetic correlation? Genetics Selection Evolution, 46, 79.

Blows, M.W. \& Hoffmann, A.A. (2005). A reassessment of genetic limits to evolutionary change. Ecology, 86, 1371-1384.

Bonebrake, T.C. \& Mastradrea, M.D. (2010). Tolerance adaptation and precipitation changes complicate latitudinal patterns of climate change impacts. Proceedings of the National Academy of Science USA, 107, 12581-12586.

Bridle, J.R., Gavaz, S. \& Kennington, W.J. (2009). Testing limits to adaptation along altitudinal gradients in rainforest Drosophila. Proceedings of the Royal Society B: Biological Sciences, 276, 1507-1515.

Bridle, J.R. \& Vines, T.H. (2007). Limits to evolution at range margins: when and why does adaptation fail? Trends in Ecology and Evolution, 22, 140 - 147.

Careau, V., Wolak, M.E., Carter, P.A. \& Garland Jr., T. (2015). Evolution of the additive genetic variance - covariance matrix under continuous directional selection on a complex behavioural phenotype. Proceedings of the Royal Society B: Biological Sciences, 282, 20151119.

Charmantier, A. \& Garant, D. (2005). Environmental quality and evolutionary potential: lessons from wild populations. Proceedings of the Royal Society B: Biological Sciences, 272, 1415-1425.

Churchill, E.R., Bridle, J.R. \& Thom, M.D. (2020). Spatially clustered resources increase male aggregation and mating duration in Drosophila melanogaster. Animal Behaviour, 169, 45-50.

Churchill, E.R., Dytham, C., Bridle, J.R. \& Thom, M.D. (2021). Social and physical environment independently affect oviposition decisions in Drosophila melanogaster. bioRxiv.

Cocciardi, J.M., O'Brien, E.K., Hoskin, C.J., Stoetzel, H. \& Higgie, M. (2021). The predictive potential of key adaptation parameters and proxy fitness traits between benign and stressful thermal environments. bioRxiv.

Darwin, C. (1859). On the Origin of Species by Means of Natural Selection. John Murray, London. 
Deutsch, C.A., Tewksbury, J.J., Huey, R.B., Sheldon, K.S., Ghalambor, C.K., Haak, D.C. et al. (2008). Impacts of climate warming on terrestrial ectotherms across latitude. Proceedings of the National Academy of Science USA, 105, 6668 - 6672.

Endler, J.A. (1986). Natural Selection in the Wild. Princeton University Press.

Etterson, J.R. (2004). Evolutionary potential of Chamaecrista fasciculata in relation to climate change I. Clinal patterns of selection along an environmental gradient in the great plains. Evolution, 58, 1446 - 1458.

Falconer, D.S. \& Mackay, T.F.C. (1996). Introduction to quantitative genetics, 4th edition. Longman, Essex.

Gienapp, P., Teplitsky, C., Alho, J.S., Mills, J.A. \& Merilä, J. (2008). Climate change and evolution: disentangling environmental and genetic responses. Molecular Ecology, 17, 167 - 178.

Griffiths, J.A., Schiffer, M. \& Hoffmann, A.A. (2005). Clinal variation and laboratory adaptation in the rainforest species Drosophila birchii for stress resistance, wing size, wing shape and development time. Journal of Evolutionary Biology, 18, 213 222.

Hadfield, J.D. (2010). MCMC Methods for Multi-Response Generalized Linear Mixed Models: The MCMCglmm R Package. Journal of Statistical Software, 33, 1-22.

Hallas, R., Schiffer, M. \& Hoffmann, A.A. (2002). Clinal variation in Drosophila serrata for stress resistance and body size. Genetics Research, 79, 141 - 148.

Hansen, T.F. \& Houle, D. (2008). Measuring and comparing evolvability and constraint in multivariate characters. Journal of Evolutionary Biology, 21, 1201-1219.

Hayward, A.D., Pemberton, J.M., Berenos, C., Wilson, A.J., Pilkington, J.G. \& Kruuk, L.E. (2018). Evidence for selection-by-environment but not genotype-byenvironment interactions for fitness-related traits in a wild mammal population. Genetics, 208, 349-364.

Hayward, A.D., Pemberton, J.M., Berenos, C., Wilson, A.J., Pilkington, J.G. \& Kruuk, L.E.B. (2017). Evidence for selection-by-environment but not genotype-byenvironment interactions for fitness-related traits in a wild mammal population. Genetics.

Hendry, A.P., Taylor, E.B. \& McPhail, J.D. (2002a). Adaptive divergence and the balance between selection and gene flow: lake and stream stickleback in the Misty system. Evolution, 56, 1199 - 1216.

Hendry, A.P., Taylor, E.B. \& McPhail, J.D. (2002b). Adaptive divergence and the balance between selection and gene flow: lake and stream stickleback in the Misty system. Evolution, 56, 1199-1216. 
Hereford, J., Hansen, T.F. \& Houle, D. (2004). Comparing strengths of directional selection: how strong is strong? Evolution, 58, 2133 - 2143.

Hoffmann, A.A., Anderson, A. \& Hallas, R. (2002). Opposing clines for high and low temperature resistance in Drosophila melanogaster. Ecology Letters, 5, 614 - 618.

Hoffmann, A.A., Hallas, R.J., Dean, J.A. \& Schiffer, M. (2003). Low potential for climatic stress adaptation in a rainforest Drosophila species. Science, 301, 100-102.

Hoffmann, A.A. \& Merilä, J. (1999). Heritable variation and evolution under favourable and unfavourable conditions. Trends in Ecology \& Evolution, 14, 96-101.

Hoffmann, A.A. \& Sgrò, C.M. (2011). Climate change and evolutionary adaptation. Nature, 470, 479 - 485.

Holman, L. \& Jacomb, F. (2017). The effects of stress and sex on selection, genetic covariance, and the evolutionary response. Journal of evolutionary biology, 30 , 1898-1909.

Husby, A., Visser, M.E. \& Kruuk, L.E. (2011). Speeding up microevolution: the effects of increasing temperature on selection and genetic variance in a wild bird population. PLoS biology, 9, e1000585.

Jeffs, C.T., Terry, J.C.D., Higgie, M., Jandová, A., Konvičková, H., Brown, J.J. et al. (2021). Molecular analyses reveal consistent food web structure with elevation in rainforest Drosophila-parasitoid communities. Ecography, 44, 403-413.

Kellermann, V., van Heerwaarden, B., Sgro, C.M. \& Hoffmann, A.A. (2009). Fundamental evolutionary limits in ecological traits drive Drosophila species distributions. Science, 325, 1244 - 1246.

Kellermann, V.M., Van Heerwaarden, B., Hoffmann, A.A. \& Sgrò, C.M. (2006). Very low additive genetic variance and evolutionary potential in multiple populations of two rainforest Drosophila species. Evolution, 60, 1104 - 1108.

Kingsolver, J.G., Hoekstra, H.E., Hoekstra, J.M., Berrigan, D., Vignieri, S.N., Hill, C.E. et al. (2001). The strength of phenotypic selection in natural populations. The American Naturalist, 157, 245-261.

Kruuk, L.E.B. (2004). Estimating genetic parameters in natural populations using the 'animal model'. Philosophical Transactions of the Royal Society of London. Series B, Biological Sciences, 359, 873 - 890.

Kruuk, L.E.B., Slate, J., Pemberton, J.M., Brotherstone, S., Guinness, F. \& Clutton-Brock, T. (2002). Antler size in red deer: Heritability and selection but no evolution. Evolution, 56, 1683-1695. 
Kruuk, L.E.B., Slate, J. \& Wilson, A.J. (2008). New answers for old questions: the evolutionary quantitative genetics of wild animal populations. Annual Review of Ecology, Evolution and Systematics, 39, 525 - 548.

Lande, R. (1979). Quantitative genetic analysis of multivariate evolution, applied to brain: body size allometry. Evolution, 33, 402-416.

Lenormand, T. (2002). Gene flow and the limits to natural selection. Trends in Ecology \& Evolution, 17, 183-189.

Linnen, C.R. \& Hoekstra, H.E. (2009). Measuring natural selection on genotypes and phenotypes in the wild. Cold Spring Harbour Symposium on Quantitative Biology, 74, 155-168.

Logan, M.L., Cox, R.M. \& Calsbeek, R. (2014). Natural selection on thermal performance in a novel thermal environment. Proceedings of the National Academy of Science $U S A, 111,14165$ - 14169.

Louthan, A.M., Doak, D.F. \& Angert, A.L. (2015). Where and when do species interactions set range limits? Trends in Ecology \& Evolution, 30, 780 - 792.

Lush, J. (1937). Animal Breeding Plans. Iowa State College Press, Ames, Iowa.

Malek, H.L. \& Long, T.A. (2020). On the use of private versus social information in oviposition site choice decisions by Drosophila melanogaster females. Behavioral Ecology, 31, 739-749.

Mousseau, T.A. \& Roff, D.A. (1987). Natural selection and the heritability of fitness components. Heredity, 59, 181-198.

Mousseau, T.A., Sinervo, B. \& Endler, J.A. (2000). Adaptive genetic variation in the wild. Oxford University Press, New York.

Nadeau, C.P., Urban, M.C. \& Bridle, J.R. (2017). Climates past, present, and yet-to-come shape climate change vulnerabilities. Trends in Ecology \& Evolution, 32, 786 800.

O'Brien, E.K., Higgie, M., Reynolds, A., Hoffmann, A.A. \& Bridle, J.R. (2017). Testing for local adaptation and evolutionary potential along altitudinal gradients in rainforest Drosophila: beyond laboratory estimates. Global change biology, 23, 1847-1860.

O’Brien, E.K., Higgie, M., Jeffs, C.T., Hoffmann, A.A., Hrček, J., Lewis, O.T. et al. (2020). Fitness effects of competition within and between species change across species' ranges, and reveal limited local adaptation in rainforest Drosophila. bioRxiv, 395624.

Paquette, A. \& Hargreaves, A.L. (2021). Biotic interactions are more important at species' warm vs. cool range-edges: a synthesis. bioRxiv. 
Pemberton, J.M. (2010). Evolution of quantitative traits in the wild: mind the ecology. Philosophical Transactions of the Royal Society of London. Series B, Biological Sciences, 365, 2431 - 2438.

Postma, E., Visser, J. \& Van Noordwijk, A.J. (2007). Strong artificial selection in the wild results in predicted small evolutionary change. Journal of Evolutionary Biology, $20,1823-1832$.

Quinn, J.L., Patrick, S.C., Bouwhuis, S., Wilkin, T.A. \& Sheldon, B.C. (2009). Heterogeneous selection on a heritable temperament trait in a variable environment. Journal of Animal Ecology, 78, 1203 - 1215.

$\mathrm{R}$ CoreTeam (2020). $R$ : A language and environment for statistical computing. $\mathrm{R}$ Foundation for Statistical Computing, Vienna, Austria.

Ramakers, J.J., Culina, A., Visser, M.E. \& Gienapp, P. (2018). Environmental coupling of heritability and selection is rare and of minor evolutionary significance in wild populations. Nature ecology \& evolution, 2, 1093-1103.

Rowiński, P.K. \& Rogell, B. (2017). Environmental stress correlates with increases in both genetic and residual variances: A meta - analysis of animal studies. Evolution, 71, 1339-1351.

RStudio Team (2021). RStudio: Integrated Development Environment for R.

Sala, O.E., Chapin, F.S., Armesto, J.J., Berlow, E., Bloomfield, J., Dirzo, R. et al. (2000). Global biodiversity scenarios for the year 2100. Science, 287, 1770-1774.

Saxon, A.D., O'Brien, E.K. \& Bridle, J.R. (2018). Temperature fluctuations during development reduce male fitness and may limit adaptive potential in tropical rainforest Drosophila. Journal of Evolutionary Biology, 31, 405 - 415.

Schiffer, M., Kennington, W.J., Hoffmann, A.A. \& Blacket, M.J. (2007). Lack of genetic structure among ecologically adapted populations of an Australian rainforest Drosophila species as indicated by microsatellite markers and mitochondrial DNA sequences. Molecular Ecology, 16, 1687 - 1700.

Siepielski, A.M., DiBattista, J.D. \& Carlson, S.M. (2009). It's about time: the temporal dynamics of phenotypic selection in the wild. Ecology Letters, 12, 1261 - 1276.

Siepielski, A.M., Gotanda, K.M., Morrissey, M.B., Diamond, S.E., DiBattista, J.D. \& Carlson, S.M. (2013). The spatial patterns of directional phenotypic selection. Ecology Letters, 16, 1382 - 1392.

Siepielski, A.M., Morrissey, M.B., Buoro, M., Carlson, S.M., Caruso, C.M., Clegg, S.M. et al. (2017). Precipitation drives global variation in natural selection. Science, 355, 959 - 962. 
Sørensen, J.G., Norry, F.M., Scannapieco, A.C. \& Loeschcke, V. (2005). Altitudinal variation for stress resistance traits and thermal adaptation in adult Drosophila buzzatii from the New World. Journal of Evolutionary Biology, 18, 829 - 837.

Urban, M.C. (2015). Accelerating extinction risk from climate change. Science, 348, 571 573.

Urban, M.C., Bocedi, G., Hendry, A.P., Mihoub, J.-B., Pe'er, G., Singer, A. et al. (2016). Improving the forecast for biodiversity under climate change. Science, 353, aad8466.

van Heerwaarden, B., Kellermann, V., Schiffer, M., Blacket, M., Sgrò, C.M. \& Hoffmann, A.A. (2009). Testing evolutionary hypotheses about species borders: patterns of genetic variation towards the southern borders of two rainforest Drosophila and a related habitat generalist. Proceedings of the Royal Society B: Biological Sciences, 276,1517 - 1526.

Van Heerwaarden, B. \& Sgrò, C.M. (2014). Is adaptation to climate change really constrained in niche specialists? Proceedings of the Royal Society B: Biological Sciences, 281, 20140396.

Visscher, P.M. (1998). On the sampling variance of intraclass correlations and genetic correlations. Genetics, 149, 1605 - 1614.

Walsh, B. \& Blows, M.W. (2009). Abundant genetic variation + strong selection = multivariate genetic constraints: a geometric view of adaptation. Annual Review of Ecology, Evolution and Systematics, 40, 41 - 59.

Wessels, F.J., Kristal, R., Netter, F., Hatle, J.D. \& Hahn, D.A. (2011). Does it pay to delay? Flesh flies show adaptive plasticity in reproductive timing. Oecologia, 165, 311320 .

Wilson, A.J., Coltman, D.W., Pemberton, J.M., Overall, A.D.J., Byrne, K.A. \& Kruuk, L.E.B. (2005). Maternal genetic effects set the potential for evolution in a free-living vertebrate population. Journal of Evolutionary Biology, 18, 405-414.

Wilson, A.J., Pemberton, J.M., Pilkington, J., Coltman, D.W., Mifsud, D., Clutton-Brock, T.H. et al. (2006). Environmental coupling of selection and heritability limits evolution. PLoS biology, 4, e216.

Wilson, A.J., Pemberton, J.M., Pilkington, J.G., Clutton-Brock, T.H., Coltman, D.W. \& Kruuk, L.E.B. (2007). Quantitative genetics of growth and cryptic evolution of body size in an island population. Evolutionary Ecology, 21, 337 - 356.

Wilson, A.J., Reale, D., Clements, M.N., Morrissey, M.M., Postma, E., Walling, C.A. et al. (2010). An ecologists guide to the animal model. Journal of Animal Ecology, 79, $13-26$. 
Wood, C.W. \& Brodie III, E.D. (2015). Environmental effects on the structure of the G - matrix. Evolution, 69, 2927-2940.

Wood, C.W. \& Brodie III, E.D. (2016). Evolutionary response when selection and genetic variation covary across environments. Ecology Letters, 19, 1189-1200. 


\section{(A) Cold tolerance}
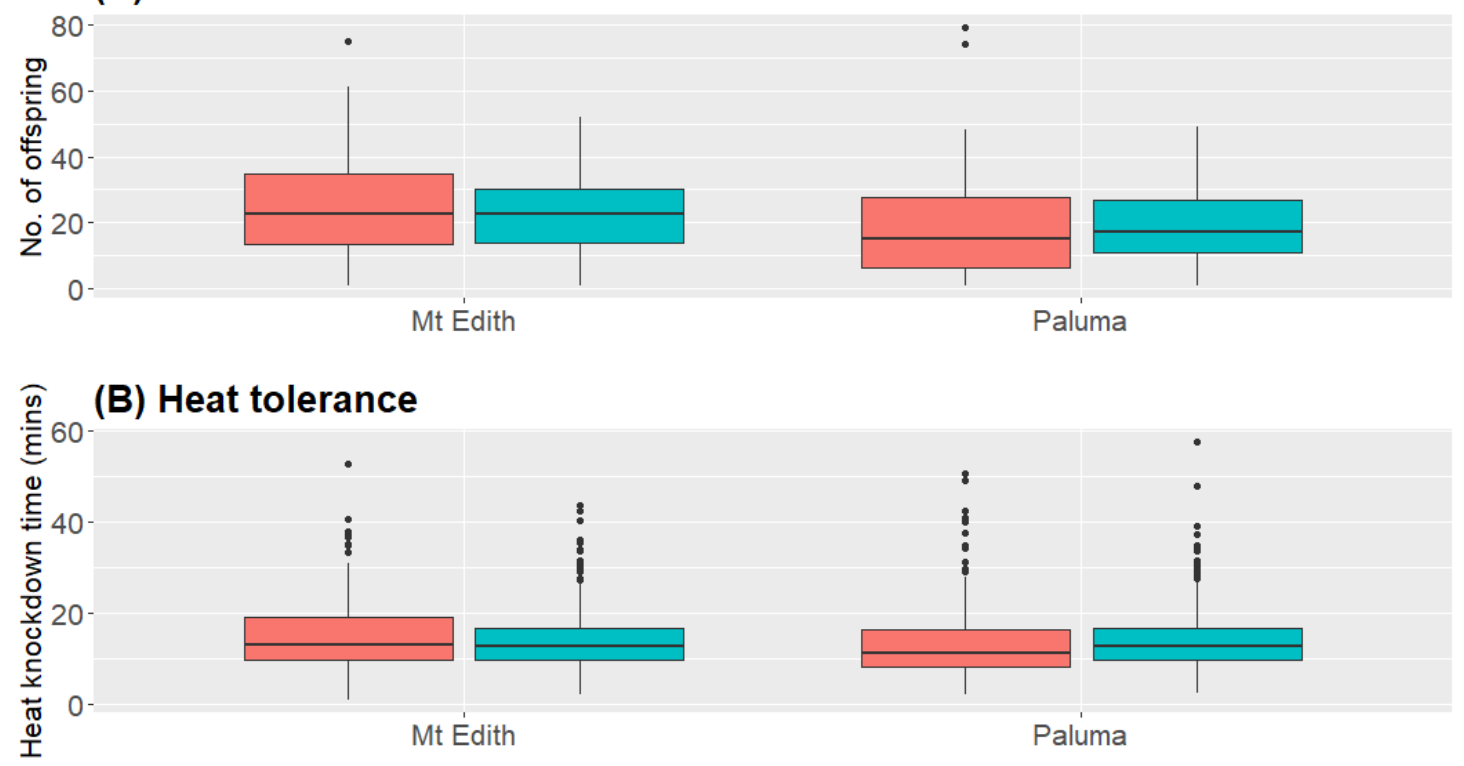

Source elevation

\section{(C) Body size}

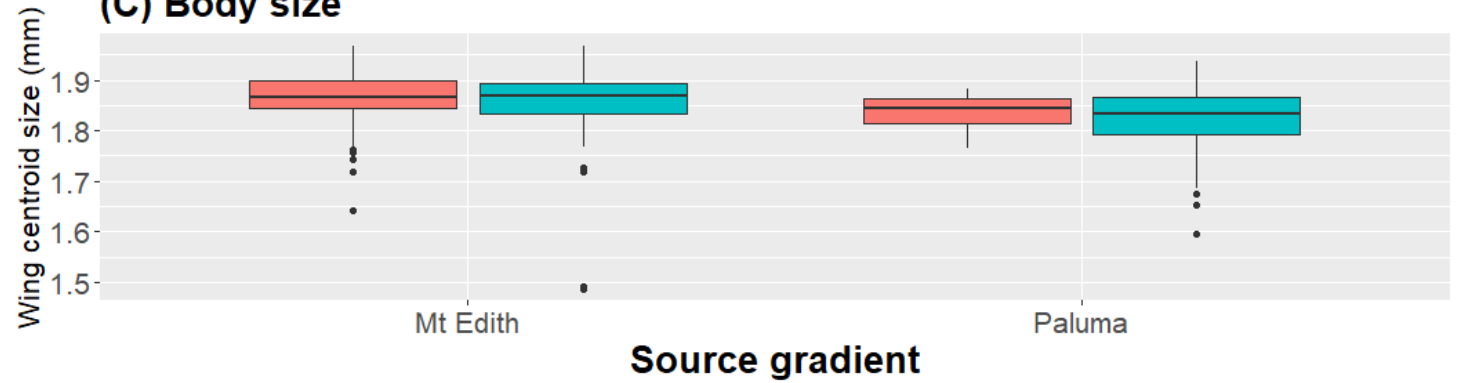

Figure 1. Box plots showing trait values of $D$. birchii from high and low elevation populations at the Mt Edith and Paluma gradients, based on line means. Traits shown are: (A) cold tolerance, (B) heat tolerance and $(C)$ body size. The dark black line is the trait median, the edges of the box are the upper and lower quartiles and the whiskers show the range of the data, with outliers shown as dots. D. birchii from Mt Edith had significantly higher cold tolerance and larger body size than those from Paluma (see Table 1), but we did not detect divergence between high and low elevation populations within gradients for any of the traits. 
Mt Edith
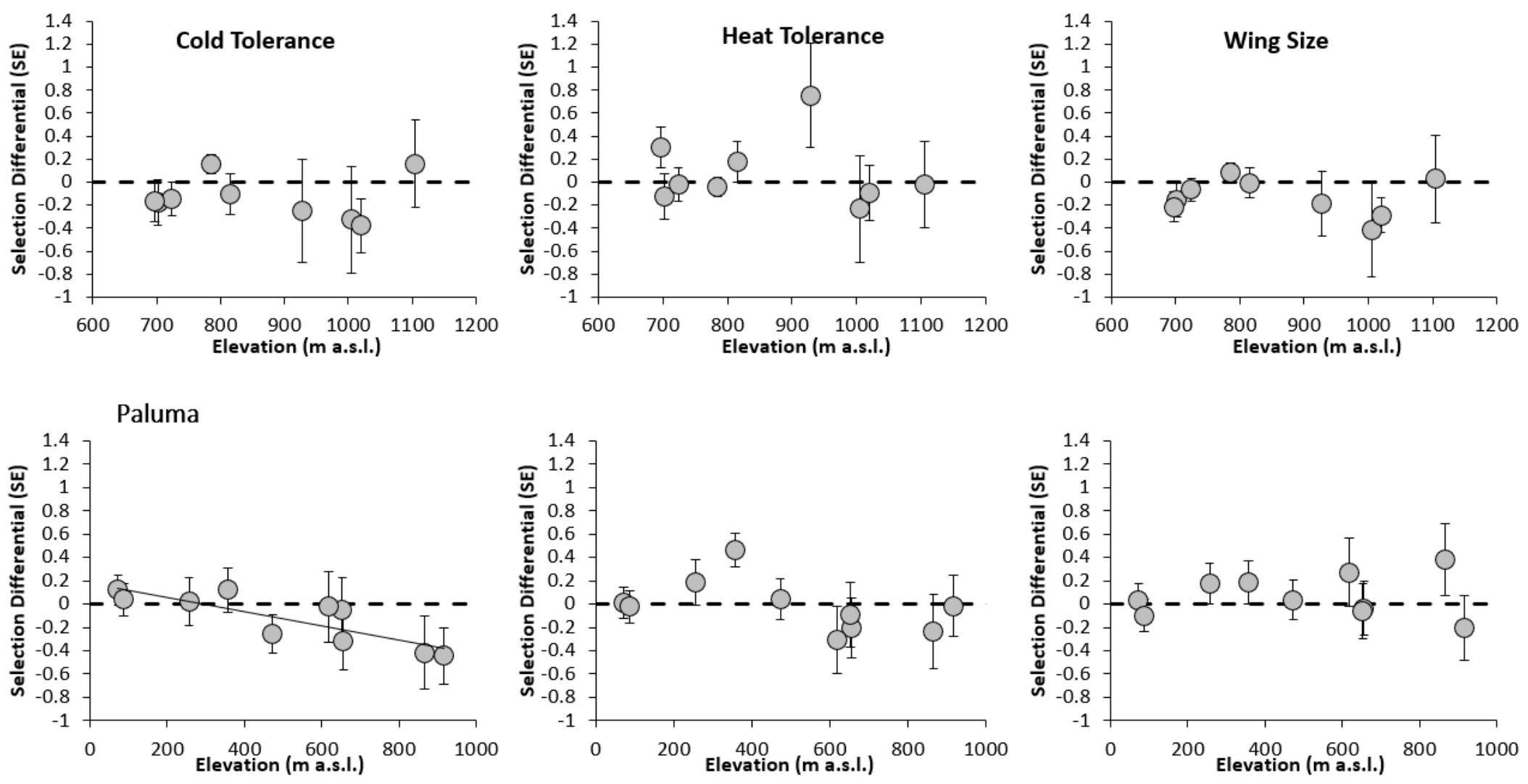

Figure 2. Selection differentials for three traits of Drosophila birchii (CT = cold tolerance, $\mathrm{HT}=$ heat tolerance, WS = wing size), estimated from the coefficients of regressions of trait mean on relative fitness at each site along two elevation gradients used in caged transplant experiments: Mt Edith (top row) and Paluma (bottom row). Error bars are standard errors of the regression coefficients at each site. We tested for a linear relationship of selection differential with elevation along each gradient for each trait. This was only detected for cold tolerance at Paluma, where selection differentials were increasingly negative at higher elevations $\left(R^{2}=0.691, F_{1,8}=17.9, P=0.0029\right)$. 


\section{Mt Edith}

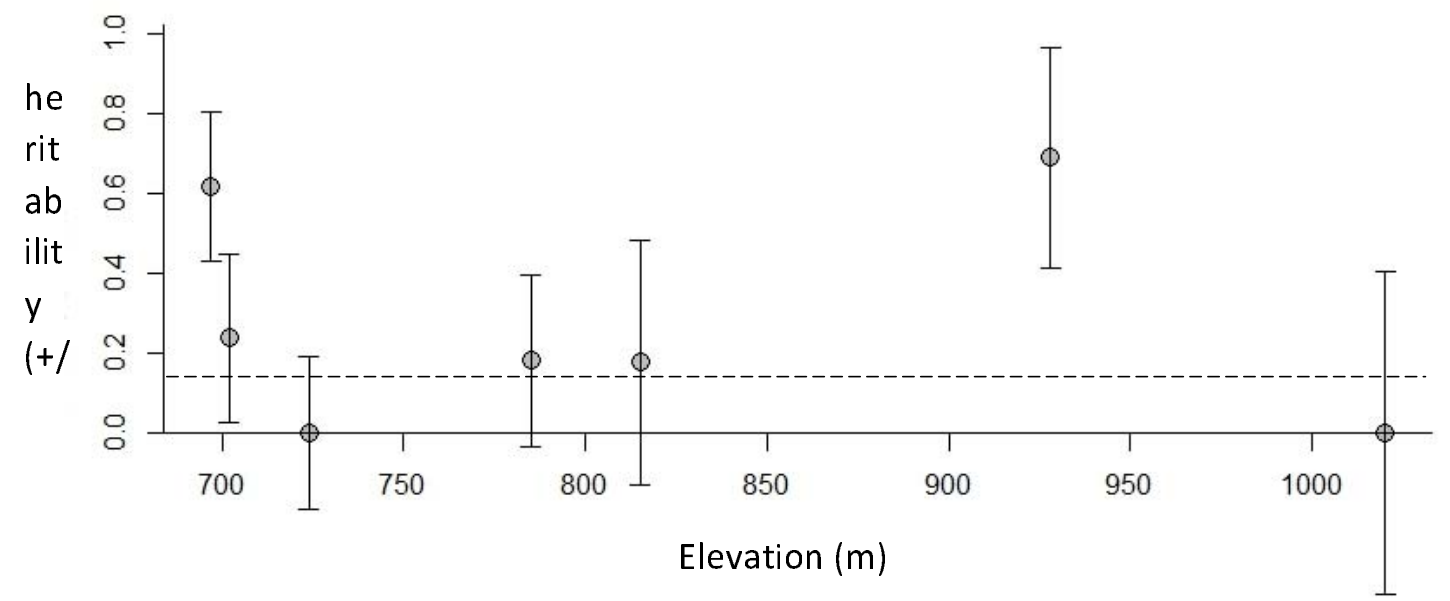

\section{Paluma}

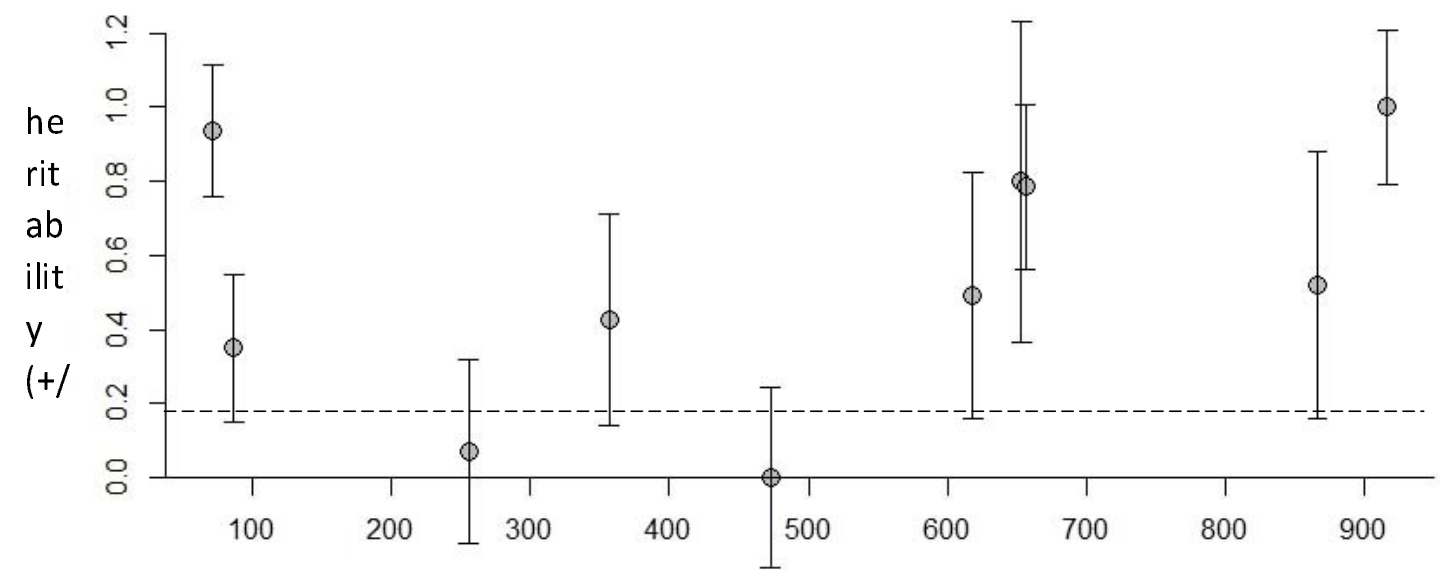

Elevation (m)

Figure 3: Heritability of $D$. birchii wing size, estimated as twice the slope of a regression of standardised wing size of female offspring emerging from field cages on standardised wing size of surviving lab-reared mothers in each cage, at each transplant site along the Mt Edith (top) and Paluma (bottom) gradients. Error bars are standard errors of the slope estimated from the regression analysis. Dashed lines indicate estimates of heritability of this trait obtained from the laboratory crossing design at each gradient (see Table 2). A linear regression of field heritability of wing size on elevation was not significant at either gradient (Mt Edith: $R^{2}=0.000, F_{1,5}=0.039, P=$ 0.850; Paluma: $R^{2}=0.003, F_{1,8}=1.03, P=0.340$ ). 

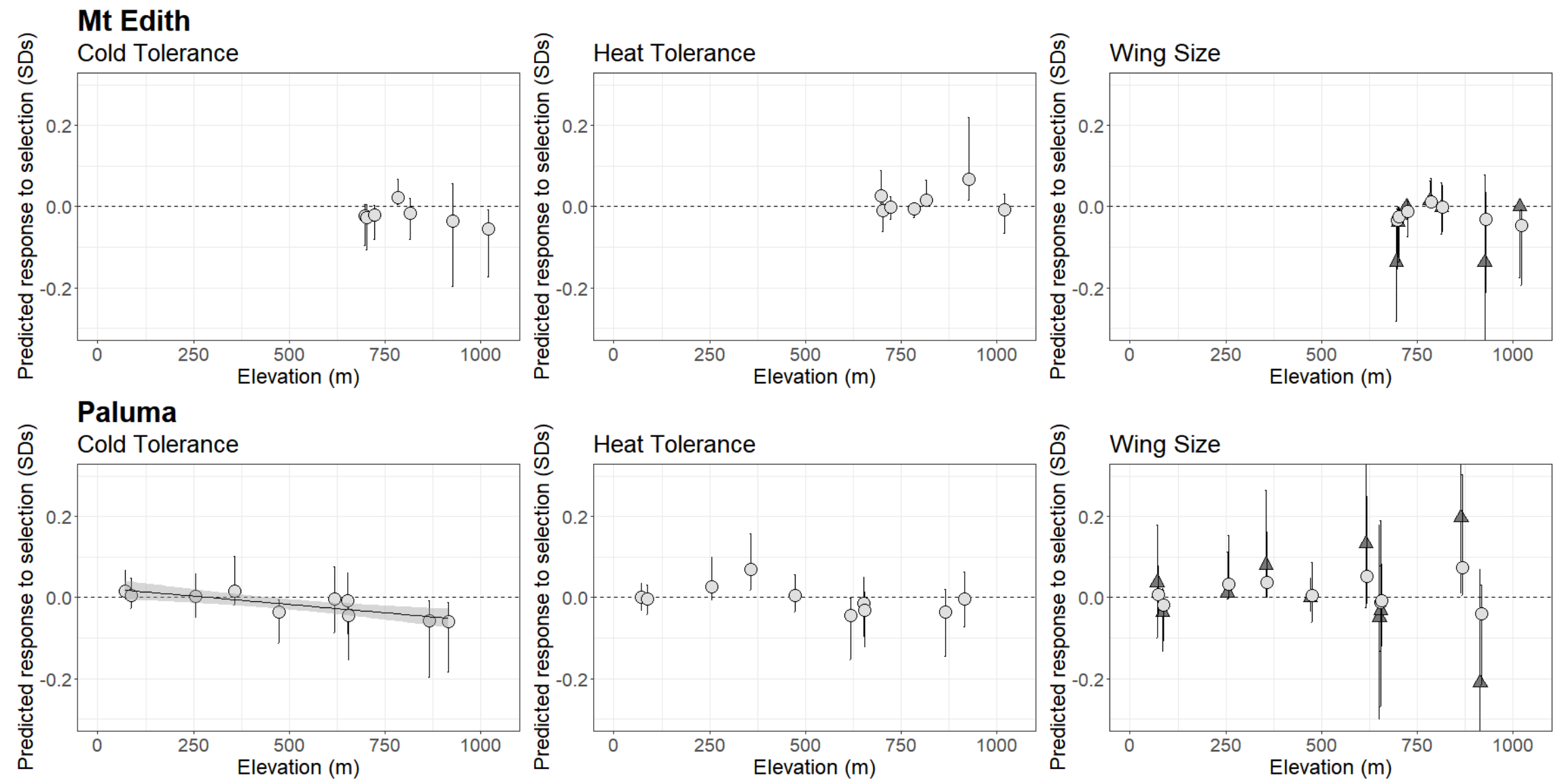

Figure 4. Predicted response to selection (in standard deviations (SDs) per generation) on cold tolerance, heat tolerance and wing size at sites along the two elevation gradients used in caged transplant experiments: Mt Edith (top) and Paluma (bottom). Predicted response to selection at each site was calculated using the breeders' equation, as the product of the selection differential $S$ (see Figure 2; Table S2) and the trait heritability $h^{2}$ calculated in the lab (all traits; light grey circles; see Table 2) or the field (wing size only; dark grey triangles; see Table S3). 


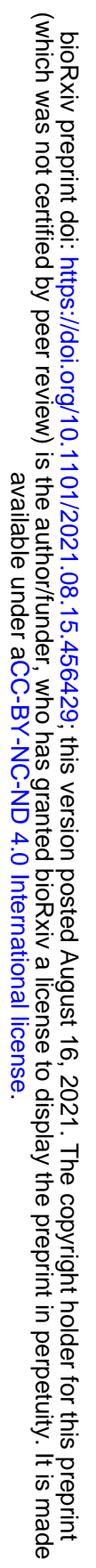


Table 1. Results of linear mixed models testing for variation in (a) cold tolerance, (b) heat tolerance and (c) body size of Drosophila birchii sourced from high and low elevation populations at the Danbulla and Paluma gradients. For each trait, ANOVA tables report the fixed effects of source gradient, elevation and their interaction. Variance due to the random effects of rearing vial ("Vial")(all traits) and batch (heat tolerance only) are also shown.

(a) Cold tolerance

Variance components

\begin{tabular}{|l|l|l|l|l|}
\hline Random effect & Variance & \% variance & $\left.\mathbf{X}^{\mathbf{2}} \mathbf{1} \mathbf{~ d f}\right)$ & $\boldsymbol{P}$ \\
\hline Vial & 57.84 & 30.7 & 14.52 & 0.0001 \\
\hline Residual & 130.78 & 69.3 & & \\
\hline
\end{tabular}

(b) Heat tolerance

ANOVA table of fixed effects

\begin{tabular}{|l|l|l|l|l|l|}
\hline Fixed effect & SS & Num df & Den df & F-value & $\boldsymbol{P}$ \\
\hline Gradient & 97.3 & 1 & 554.5 & 1.79 & 0.182 \\
\hline Elevation & 115.3 & 1 & 402.9 & 2.12 & 0.145 \\
\hline $\begin{array}{l}\text { Gradient } x \\
\text { Elevation }\end{array}$ & 10.9 & 1 & 450.5 & 0.20 & 0.655 \\
\hline
\end{tabular}

Variance components

\begin{tabular}{|l|l|l|l|l|}
\hline Random effect & Variance & \% variance & $\mathbf{X}^{\mathbf{2}}(\mathbf{1} \mathbf{d f})$ & $\boldsymbol{P}$ \\
\hline Vial & 2.15 & 3.1 & 0.81 & 0.369 \\
\hline Batch & 12.01 & 17.5 & 63.41 & $1.68 \times 10^{-15}$ \\
\hline Residual & 54.50 & 79.4 & & \\
\hline
\end{tabular}

(c) Body size

ANOVA table of fixed effects

\begin{tabular}{|l|l|l|l|l|l|}
\hline Fixed effect & SS & Num df & Den df & F-value & $P$ \\
\hline
\end{tabular}


bioRxiv preprint doi: https://doi.org/10.1101/2021.08.15.456429; this version posted August 16, 2021. The copyright holder for this preprint (which was not certified by peer review) is the author/funder, who has granted bioRxiv a license to display the preprint in perpetuity. It is made available under aCC-BY-NC-ND 4.0 International license.

\begin{tabular}{|l|l|l|l|l|l|}
\hline Gradient & 0.0344 & 1 & 57.2 & 15.01 & 0.0003 \\
\hline Elevation & 0.0038 & 1 & 88.8 & 1.68 & 0.199 \\
\hline $\begin{array}{l}\text { Gradient } x \\
\text { Elevation }\end{array}$ & 0.00004 & 1 & 88.8 & 0.02 & 0.896 \\
\hline
\end{tabular}

Variance components

\begin{tabular}{|l|l|l|l|l|}
\hline Random effect & Variance & \% variance & $\mathbf{X}^{\mathbf{2}} \mathbf{( 1 \mathbf { d f } )}$ & $\boldsymbol{P}$ \\
\hline Vial & $7.76 \times 10^{-4}$ & 25.3 & 45.42 & $1.59 \times 10^{-11}$ \\
\hline Residual & $2.29 \times 10^{-3}$ & 74.7 & & \\
\hline
\end{tabular}


Table 2. (a) Heritabilities (on the diagonal, in bold) of traits and genetic correlations (below the diagonal, in italics) among traits and (b) Maternal effect variance as a proportion of total phenotypic variance (on the diagonal, in bold) of traits and maternal effect correlations (below the diagonal, in italics) among traits for $D$. birchii from Danbulla and Paluma, with upper and lower $95 \%$ Highest Posterior Density (HPD) intervals for each estimate. CT=Cold Tolerance; HT=Heat Tolerance; WS=Wing Size. Note that wing size was measured on offspring from different mothers to the other two traits, therefore maternal effect correlations were not estimated for wing size.

(a) Heritabilities and genetic correlations

\begin{tabular}{|c|c|c|c|c|c|c|c|}
\hline \multirow[b]{2}{*}{ Trait } & \multicolumn{3}{|c|}{ Mt Edith } & \multicolumn{4}{|c|}{ Paluma } \\
\hline & CT & HT & WS & Trait & CT & HT & WS \\
\hline CT & $\begin{array}{l}0.145 \\
(0.06, \\
0.28)\end{array}$ & & & CT & $\begin{array}{l}0.136 \\
(0.06, \\
0.27)\end{array}$ & & \\
\hline HT & $\begin{array}{l}0.040 \\
(-0.41 \\
0.57)\end{array}$ & $\begin{array}{l}0.089 \\
(0.05 \\
0.19)\end{array}$ & & HT & $\begin{array}{l}0.048 \\
(-0.44 \\
0.49)\end{array}$ & $\begin{array}{l}0.150 \\
(0.06 \\
0.26)\end{array}$ & \\
\hline WS & $\begin{array}{l}0.047 \\
(-0.60 \\
0.54)\end{array}$ & $\begin{array}{l}0.097 \\
(-0.52 \\
0.51)\end{array}$ & $\begin{array}{l}0.156 \\
(0.06, \\
0.44)\end{array}$ & WS & $\begin{array}{l}0.126 \\
(-0.57, \\
0.58)\end{array}$ & $\begin{array}{l}0.054 \\
(-0.68 \\
0.47)\end{array}$ & $\begin{array}{c}0.193 \\
(0.07, \\
0.44)\end{array}$ \\
\hline
\end{tabular}

(b) Maternal effect variance and correlations

\begin{tabular}{|c|c|c|c|c|c|c|c|}
\hline & \multicolumn{3}{|c|}{ Mt Edith } & & \multicolumn{3}{|c|}{ Paluma } \\
\hline Trait & CT & HT & WS & Trait & CT & HT & WS \\
\hline \multirow[t]{2}{*}{ CT } & 0.149 & & & CT & 0.139 & & \\
\hline & $\begin{array}{l}(0.08, \\
0.25)\end{array}$ & & & & $\begin{array}{l}(0.08, \\
0.24)\end{array}$ & & \\
\hline \multirow[t]{2}{*}{ HT } & 0.202 & 0.104 & & HT & 0.030 & 0.090 & \\
\hline & $\begin{array}{l}(-0.30 \\
0.49)\end{array}$ & $\begin{array}{l}(0.05, \\
0.16)\end{array}$ & & & $\begin{array}{l}(-0.38 \\
0.41)\end{array}$ & $\begin{array}{l}(0.05, \\
0.15)\end{array}$ & \\
\hline \multirow[t]{2}{*}{ WS } & & & 0.109 & ws & & & 0.140 \\
\hline & & & $\begin{array}{l}(0.06, \\
0.30)\end{array}$ & & & & $\begin{array}{l}\text { (0.07, } \\
0.29)\end{array}$ \\
\hline
\end{tabular}

